\title{
A Study of Posterior Segment Pathology in Cataractous Eyes Using B-scan Ultrasonography
}

\author{
Mohammed Abdalla Shakour ${ }^{1}$, Caroline E. Ayad ${ }^{1}$, Samih Kajoak ${ }^{2}$, \\ Hamid Osman ${ }^{2 *}$, E. Rahim ${ }^{2}$ \\ ${ }^{1}$ College of Medical Radiological Sciences, Sudan University of Science and Technology, Khartoum, Sudan \\ ${ }^{2}$ Taif University, College of Medical Applied Sciences, Department of Diagnostic Radiology Sciences, \\ Taif, Saudi Arabia
}

\begin{abstract}
Background: Ultrasonography (US) is a valuable diagnostic modality for detecting posterior segment eye diseases (PSEDs) in patients with cataractous eyes. This imaging modality can better facilitate planning surgery and predicting prognosis. The purpose of this study was to determine the role of B-scan US in evaluating posterior segment abnormalities of eyes in cataract patients pre-operatively to limit complications and visual impairment.

Methods and Results: A prospective, cross-sectional study to assess cataracts by US, as well as to detect posterior segment abnormalities of eyes in cataract patients, was conducted in Sudan at Albasar International Foundation (Makah Eye Complex in Omdurman) between December 2018 and December 2019.

All patients ( $n=380 ; 48 \%$ males and $52 \%$ females; a mean age of $63 \pm 12.57$ years) with non-visualization of the fundus, regardless of age and gender, were involved and were assumed to have orbital pathologies. Patients with high-risk extrusion of intraocular contents and a previous history of ocular surgery were excluded from the present study. All patients with dense cataracts were evaluated using standard US machines (Echoscan US-4000; NIDEK CO., LTD.) equipped with a real-time highfrequency probe.

The typical age of the patients complaining of cataracts was between 61 and 70 years, which constituted $35 \%$ of cases, followed by age $>70$ years, which constituted $24.2 \%$ of cases. About $47.63 \%$ patients had ocular pathology in the posterior segment of the eye. The most common PSEDs were vitreous abnormalities (46.2\%). Among vitreous abnormalities, most abnormal eyes had vitreous changes $(36.1 \%)$, followed by vitreous detachment $(3.7 \%) ; 1.3 \%$ of patients had retinal detachment.

Conclusion: Ocular US should be the first screening modality in the evaluation of posterior segment pathologies in cataractous eyes and should be performed in cataract patients pre-operatively for better surgical planning and predicting postoperative visual prognosis.(International Journal of Biomedicine. 2021;11(1):78-81.)
\end{abstract}

Key Words: B-scan ultrasonography • cataract • posterior segment eye diseases

For citation: Shakour MA, Ayad CE, Kajoak S, Osman H, Rahim E. A Study of Posterior Segment Pathology in Cataractous Eyes Using B-scan Ultrasonography. International Journal of Biomedicine. 2021;11(1):78-81. doi:10.21103/Article11(1)_OA13

\section{Abbreviations}

PVD, posterior vitreous detachment; PSEDs, posterior segment eye diseases; RD, retinal detachment; VD, vitreous detachment; IOFB, intraocular foreign body.

\section{Introduction}

A cataract (lens opacification) is a degenerative disease of the lens and represents one of the most important causes of visual impairment. The proportion of cataract blindness among all ocular diseases ranges from 5\% in Western Europe, North America, and affluent countries to $50 \%$ in developing countries. ${ }^{(1)}$ Forecasts predict that there will be almost 115 million cases of blindness and 588 million people with moderate to severe vision impairment in 2050 (up from figures 
of 36 million and 217 million today, respectively). ${ }^{(2)}$ There are several risk factors that may be frequently associated with cataracts, such as injury, diabetes, ultraviolet irradiation, specific ocular abnormalities (e.g., uveitis), and hypertension, but the main non-modifiable risk factor is aging. Cataract surgery and insertion of an intraocular lens are highly effective, resulting in almost immediate visual rehabilitation. ${ }^{(3)}$

Among patients with vision problems, fundoscopy and slit-lamp evaluation are the basis for diagnosis. In some cases in which cataracts change posterior segment eye diseases (PSEDs), which are defined as diseases of the retina, choroid and optic nerve, ${ }^{(4)}$ Ultrasonography (US) is an indispensable diagnostic tool for evaluating and diagnosing many PSEDs. It helps the radiologist and ophthalmologist in diagnosing disease and choosing the optimal method of treatment. ${ }^{(4)} \mathrm{B}$-scan US is a powerful, non-invasive, rapid, cost-effective, and nonionizing real-time diagnostic tool that provides a good anatomic background, provides valuable diagnostic information for evaluating lesions of the posterior segment in eyes, and helps to rule out vitreous abnormalities, such as retinal, vitreous, and choroidal detachments, as well as tumors and other pathologic conditions that affect the posterior segment of the eye. ${ }^{(5)}$ These conditions are mainly attributable to the cystic nature of the eye and its surface location, which allows high-frequency probes to demonstrate normal anatomy and pathologies accurately. ${ }^{(6)}$ If the ophthalmologist and surgeon are aware of these pathologies pre-operatively, the surgical plan can be modified to better predict the visual outcome after cataract surgery and permit measures to combat anticipated complications. ${ }^{(7,8)}$

There are statistical discrepancies in previous studies with respect to the prevalence of the posterior segment of the eye among patients with cataracts. The significant variance exists among findings associated with cataracts across various populations. Vepa et al. ${ }^{(9)}$ reported that in their study posterior segment lesions occurred in 54(11.02\%) cases. Qureshi et al. ${ }^{(10)}$ reported that the prevalence of posterior segment lesions in Pakistan was $12 \%$; specifically, $3 \%$ of patients had retinal detachment (RD), $2 \%$ - posterior vitreous detachment (PVD), $3 \%$ - vitreous hemorrhage, $1.2 \%$ - posterior staphyloma, and $1 \%$ - intraocular foreign body (IOFB) ${ }^{(10)}$ Shen et al. ${ }^{(11)}$ reported vitreous detachment (VD) prevalence in a large cohort of Chinese to be $2.7 \%$.

Cataractous eyes with posterior segment pathologies have not been the subject of comprehensive reports and few studies have reported the sonographic findings related to cataracts. The purpose of this study was to determine the role of B-scan US in evaluating posterior segment abnormalities of eyes in cataract patients pre-operatively to limit complications and visual impairment.

\section{Materials and Methods}

We performed a prospective, cross $^{-}$sectional study to assess cataracts by US, as well as to detect posterior segment abnormalities of eyes in cataract patients. The study was conducted in Sudan at Albasar International Foundation (Makah Eye Complex in Omdurman) between December 2018 and December 2019.
All patients $(n=380)$ with non-visualization of the fundus, regardless of age and gender, were involved and were assumed to have orbital pathologies. Patients with high-risk extrusion of intraocular contents and a previous history of ocular surgery were excluded from the present study.

The data collection sheet was designed to collect demographic data. All registered patients underwent a preoperative evaluation protocol that included intraocular pressure, posterior synechiae, visual acuity determination, pupillary reaction, biometric measurements, and a slit-lamp test. Risk factors, such as diabetes mellitus, hypertension, corneal opacities, iris coloboma, and deviated eyes, were specifically sought and noted.

After completing an ocular examination, all patients with dense cataracts were evaluated using standard US machines (Nidek Echo scan model US-4000; company, city, state, country) equipped with a real-time high-frequency probe. The procedure was explained to the patients, who were placed in a supine position and asked to flex the neck and head slightly, then rotated to the opposite side to prevent gel spreading. The ultrasonic probe accepts linear, $7.5-10 \mathrm{MHz}$ small footprint transducers, which are suitable for this purpose. The transducer was placed over the globe of the eye with a closed eyelid after application of the gel, then anteroposterior, transverse, and longitudinal views of the B-scan, along with A-scan, were obtained for both eyes. Low- and high-gain sensitivities were selected throughout the US. Images from the machine were collected and analyzed for evaluation. We determined that significant posterior segment pathology on the US affected the post-operative visual results. Images were diagnosed by a professional sonologist and ophthalmologist.

Statistical analysis was performed using statistical software package SPSS version 16.0 (SPSS Inc, Chicago, IL).

\section{Results}

The sample consisted of 380 cataract patients (48\% males and $52 \%$ females) with a mean age of $63 \pm 12.57$ years. The patients were classified into 7 age groups. The typical age of the patients complaining of cataracts was between 61 and 70 years, which constituted $35 \%$ of cases, followed by age $>70$ years, which constituted $24.2 \%$ of cases; the other age groups are shown in Figure 1.

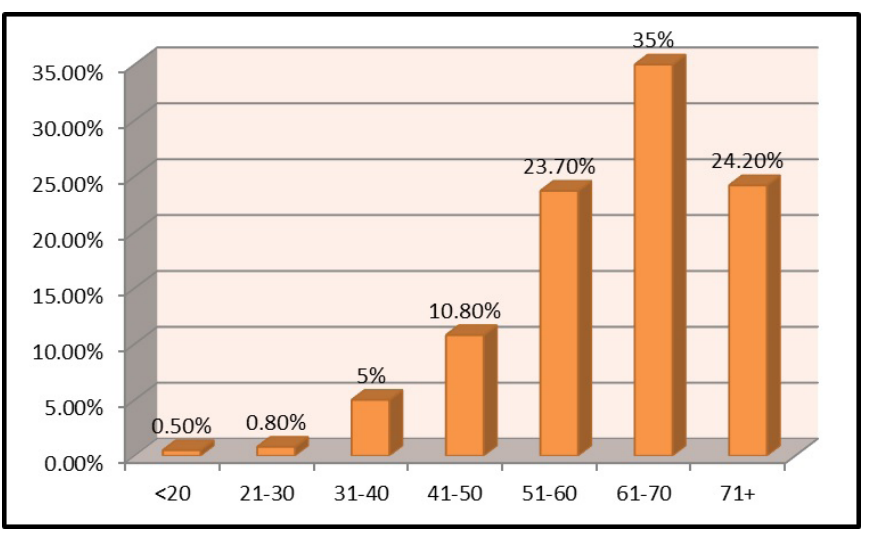

Fig. 1. Age distribution of the study sample. 
Of this total number of patients, $12.1 \%$ had diabetes mellitus and $17.9 \%$ - systemic hypertension.

About $47.63 \%$ patients had ocular pathology in the posterior segment of the eye (Table 1). The most common PSEDs were vitreous abnormalities, followed by retinal detachment.

Table 1.

Frequency distribution of pathology in the posterior segment in cataractous eyes.

\begin{tabular}{lcc}
\hline Ocular Abnormalities & No. of Abnormalities & $\%$ \\
\hline Normal & 199 & 52.37 \\
Vitreous & 176 & 46.2 \\
Retina & 5 & 1.31 \\
Choroid & 0 & 0 \\
\hline
\end{tabular}

Among vitreous abnormalities, most abnormal eyes had vitreous changes $(36.1 \%)$, followed by VD (3.7\%). Vitreous changes mean that vitreous volume was reduced, and the fibers were thickened, tortuous, and surrounded by liquid vitreous (Fig.2).

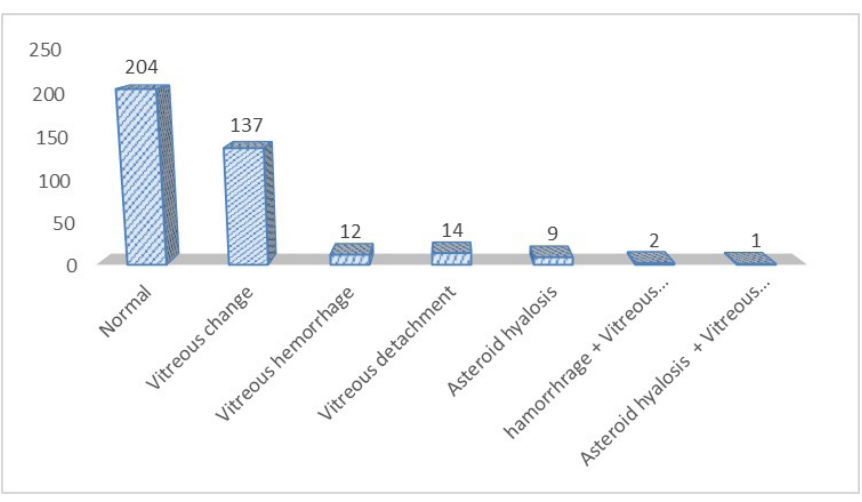

Fig. 2. Sonographic appearance of vitreous.

\section{Discussion}

Cataracts are among the most important causes of treatable blindness in developing countries, like Sudan, due to a lack of awareness and education, low-quality service, and high-cost treatment. Many patients present with advanced cataracts, which preclude fundus visualization and make clinical and ophthalmoscopic examinations difficult and minimally informative prior to cataract surgery. Under such circumstances, ophthalmic US has been shown to be an invaluable tool in the diagnosis and management of posterior segment pathology in patients with dense cataracts. Moreover, ophthalmic US is non-invasive, rapid, and cost-effective with no exposure to ionizing radiation. Thus, ophthalmic US enables a dynamic study and provides valuable diagnostic information of various ophthalmic diseases not obtainable by other imaging methods. ${ }^{(12-14)}$

Ocular abnormalities are believed to be more common in males than in females. In a study conducted by Sharma, ${ }^{(8)}$ the gender ratio was $2: 1$, respectively. In another study, by
Mcleod et al., ${ }^{(15)}$ male predominance was also reported. The current study, however, was not in agreement with the findings of previous studies; specifically, we demonstrated female predominance over males ( $52 \%$ vs. $48 \%$.

The results of the present study demonstrated that in the $35 \%$ and $24.2 \%$ of the patients who presented in the $61-70$ and $>70$-year age groups, respectively, age was statistically correlated with cataracts. In this study, $12.1 \%$ of patients had diabetes mellitus and $17.9 \%$ had hypertension. Richter et al. ${ }^{(16)}$ have confirmed that in the elderly with a history of hypertension and diabetes, those conditions were risk factors for cataracts.

In the current study, 380 cases with advanced cataracts were examined by B-scan US, which revealed that $47.63 \%$ had PSEDs. A much lower percentage of PSEDs was recorded by Salman et al. ${ }^{(17)}$ and Chanchlani et al., ${ }^{(18)}$ who reported that $9 \%$ and $8.7 \%$ of patients had PSEDs, respectively. In contrast, PSEDs were present in $77.5 \%$ of patients screened by B-mode US in cataract patients between 2005 and 2007 by Mendes et al. ${ }^{(18)}$ These statistical discrepancies concerning the prevalence of PSEDs by US may be due to exclusion and inclusion criteria in each study, examination techniques used, and the expertise of examiners, because ocular US is dependent on the skill of the operator. In addition, variations between the populations scanned in different studies are also assumed to have contributed.

In the present study, RD was identified by B-scan US in $1.31 \%$ of patients, which is less than that reported by Meenakshi et al. $-2.5 \%,{ }^{(9)}$ Qureshi et al. - 3\%, ${ }^{(10)}$ and Jain et al. - 5\%. ${ }^{(19)}$ In addition, Salman et al. ${ }^{(17)}$ reported only $0.7 \%$ patients with $\mathrm{RD}$, and RD was identified in up to $9.3 \%$ of the cases evaluated by Mendes et al. ${ }^{(18)}$ The sonographic ocular investigations in this study demonstrated that vitreous abnormalities were the most common finding (46.2\%). This finding is in agreement with previous results published by Mcleod et al., ${ }^{(15)}$ who studied 176 eyes and found that the majority of patients had vitreous abnormalities. Sharma ${ }^{(8)}$ reported similar results in another study. Vitreous changes $(36.1 \%)$, followed by vitreous hemorrhage $(3.2 \%)$ were most prevalent among patients with vitreous abnormalities. In previous studies, vitreous hemorrhage was reported in posterior segment B-scan US to range from $1 \%$ $3 \%,{ }^{(20.21)}$ which is lower than the percentage found in the present study. This disparity is assumed to be primarily attributed to the higher number of diabetic and hypertensive patients in our study, with an estimated higher prevalence of proliferative retinopathy and vitreous hemorrhage.

In conclusion, ocular US should be the first screening modality in the evaluation of posterior segment pathologies in cataractous eyes and should be performed in cataract patients pre-operatively for better surgical planning and predicting post-operative visual prognosis. Ocular US is cost-effective, rapid, and non-ionizing, which empowers US to provide useful diagnostic information about numerous ophthalmic diseases not obtained by other diagnostic imaging modalities.

*Corresponding author: Hamid Osman. Taif University, College of Medical Applied Sciences, Department of Diagnostic Radiology Sciences. Taif, Saudi Arabia.E-mail: hamidsssan@yahoo.com 


\section{Competing Interests} interests.

The authors declare that they have no competing

\section{References}

1. Pascolini D, Mariotti SP. Global estimates of visual impairment: 2010. Br J Ophthalmol. 2012 May;96(5):614-8. doi: 10.1136/bjophthalmol-2011-300539.

2. Bourne RRA, Flaxman SR, Braithwaite T, Cicinelli MV, Das A, Jonas JB, Keeffe J, Kempen JH, Leasher J, Limburg H, Naidoo K, Pesudovs K, Resnikoff S, Silvester A, Stevens GA, Tahhan N, Wong TY, Taylor HR; Vision Loss Expert Group. Magnitude, temporal trends, and projections of the global prevalence of blindness and distance and near vision impairment: a systematic review and meta-analysis. Lancet Glob Health. 2017 Sep;5(9):e888-e897. doi: 10.1016/S2214109X(17)30293-0.

3. World Health Organization. (2007)! Global Initiative for the Elimination of Avoidable Blindness : action plan 20062011. Geneva: World Health Organization. https://apps.who. int/iris/handle/10665/43754

4. Sushil K, Ruchi G. Diagnostic ultrasonography of the eye. All India Ophthalmological Society. AIOS, CME series. 2011; 24:17-9.

5. Skandesh BM, Mohan Kumar, Sreenivasa Raju N. Role of high resolution sonography (B-SCAN) in the evaluation of posterior segment lesions of the eye. International Journal of Contemporary Medicine Surgery and Radiology. 2018;3(3):C11-C16.

6. Byrne SF, Green RL. Ultrasound of the eye and orbit. 2nd ed. St. Louis: Mosby; 2002

7. Agrawal D, Ahirwal D. A study of role of B scan ultrasound in posterior segment pathology of eye. Int J Med Res Rev. 2015;3(9):969-74.

8. Sharma OP. Orbital sonography with its clinicosurgical correlation. Indian J Radiol Imaging. 2005;15:537-54.

9. Meenakshi V, Jyothirmayi T, Sree B. Role of B-Scan Ultrasonography in cataract patients in a tertiary care centre. J Evol Med Dent Sci. 2015;4(83):14525-30. doi: 10.14260/ jemds/2015/2066.
10. Qureshi MA, Laghari K. Role of B-scan ultrasonography in pre-operative cataract patients. Int J Health Sci (Qassim). 2010 Jan;4(1):31-7.

11. Shen Z, Duan X, Wang F, Wang N, Peng Y, Liu DT, Peng $\mathrm{X}$, Li S, Liang Y. Prevalence and risk factors of posterior vitreous detachment in a Chinese adult population: the Handan eye study. BMC Ophthalmol. 2013 Jul 16;13(1):33. doi: 10.1186/1471-2415-13-33.

12. Allen D, Vasavada A. Cataract and surgery for cataract. BMJ. 2006 Jul 15;333(7559):128-32. doi: 10.1136/bmj.333.7559.128. 13. De La Hoz Polo M, Torramilans Lluís A, Pozuelo Segura O, Anguera Bosque A, Esmerado Appiani C, Caminal Mitjana JM. Ocular ultrasonography focused on the posterior eye segment: what radiologists should know. Insights Imaging. 2016 Jun;7(3):351-64. doi: 10.1007/s13244-016-0471-z.

14. Chanchlani M, Chanchlani R. A study of posterior segment evaluation by B-Scan in hyper mature cataract. J Clini Exp Ophthalmol. 2016;7:1. doi: https://doi.org/10.4172/21559570.1000516.

15. McLeod D, Restori M. Ultrasonic examination in severe diabetic eye disease. Br J Ophthalmol. 1979 Aug;63(8):533-8. doi: 10.1136/bjo.63.8.533.

16. Richter GM, Torres M, Choudhury F, Azen SP, Varma R; Los Angeles Latino Eye Study Group. Risk factors for cortical, nuclear, posterior subcapsular, and mixed lens opacities: the Los Angeles Latino Eye Study. Ophthalmology. 2012 Mar;119(3):547-54. doi: 10.1016/j.ophtha.2011.09.005. 17. Salman A, Parmar P, Vanila CG, Thomas PA, Jesudasan CA. Is ultrasonography essential before surgery in eyes with advanced cataracts? J Postgrad Med. 2006 Jan-Mar;52(1):19-22. 18. Mendes MH, Betinjane AJ, Cavalcante Ade S, Cheng CT, Kara-José N. Ultrasonographic findings in patients examined in cataract detection-and-treatment campaigns: a retrospective study. Clinics (Sao Paulo). 2009;64(7):637-40. doi: 10.1590/ S1807-59322009000700005.

19. Jain A, Gauba N, Kaur I, Singh S, Jaswal H. Role of B-Scan in cataract patients. Indian J Appl Radiol. 2017;3(1):110.

20. Ali SI, Rehman H. Role of B-scan in preoperative detection of posterior segment pathologies in cataract patients. Pak J Ophthalmol. 1997;13:108-12.

21. Shaikh FU, Narsani AK, Jatoi SM, Shaikh ZA. Preoperative posterior segment evaluation by ultrasonography in dense cataract. Pak J Ophthalmol. 2009;25(3):135. 